\title{
EVALUATION OF GASTROINTESTINAL BLEEDING: CURRENT IMAGING STRATEGIES
}

Alaa Mohammed Fathy Asaad, Khaled Mohamed Morad Moghazy, *Mohamed Abdullah Sharaan, Omar Sameh El Assar, Samar Ahmed Mohamed Abdullah

Department of Radiodiagnosis, *Department of Surgery, Faculty of Medicine, Alexandria University.

\section{Introduction}

Gastrointestinal (GI) bleeding is a common cause of gastrointestina disorders, but diagnosing it can be very difficult.

Diagnosis and treatment of gastrointestinal bleeding needs participation of different specialists, different imaging techniques and selecting the most appropriate treatment from different options available. Patients with GI bleeding mostly presents to emergency department where a gastroenterologist attends and then radiologic and/or endoscopic imaging. After that interventional radiology and surgical departments are sought.

There's a wide variety of presentation of gastrointestinal bleeding ranging from asymptomatic individuals to mildly symptomatic needing only conservative treatment to severely ill patients needin immediate intervention. Therefore, it's very important for medical individuals to understand and recognize the presentation as well as the appropriate management of $\mathrm{Gl}$ bleeding.

Gl bleeding is classified according to its anatomical location into upper or lower bleeding. Upper Gastrointestinal bleeding (UGIB) is defined as bleeding proximal to ligament of Treitz.Lowe gastrointestinal bleeding (LGIB) is defined as bleeding distal to ligament of Treitz.

\section{Aim of the work}

The aim of this study was to study current imaging strategies in gastrointestinal bleeding.

\section{PATIENTS}

This study was carried out on 20 patients presented with gastrointestinal bleeding to the Radio-diagnosis Department of Alexandria Main University Hospital for management.

\section{Methods}

All patients were subjected to the following scheme :

Thorough history taking including age, onset and nature of bleeding, underlying medical conditions, prior surgeries and allergies.

Clinical examination with special focus on the patient's hemodynamic state.

Renal function was assessed with measurements of serum creatinine levels. Estimated glomerular filtration rate (eGFR) was calculated using the Cockcroft-Gault equation.

Endoscopic findings, if available, were reviewed.

Acute Gl bleeding was defined as hematemesis, melena, or hemochezia that occurred within 24 hours before CT.

Imaging evaluation including:2D Ultrasound (20 patients), routine abdomen and pelvis (7patients)andCTA (13 patients) and catheter angiography (16 patients)

\section{Results}

The twenty patients were distributed according to the final diagnosis as: six patients with GIT tumor (30\%).five with angiodysplasia.(25\%), two patients with peptic ulcer (10\%), two patients with pseudo aneurysms therapy (rectal and jeujnal extravasation (10\%). The patients presented with: 7 patients presented by hematochezia (35\%), 8 by melena (40\%), five patients with hematemesis $(25 \%)$

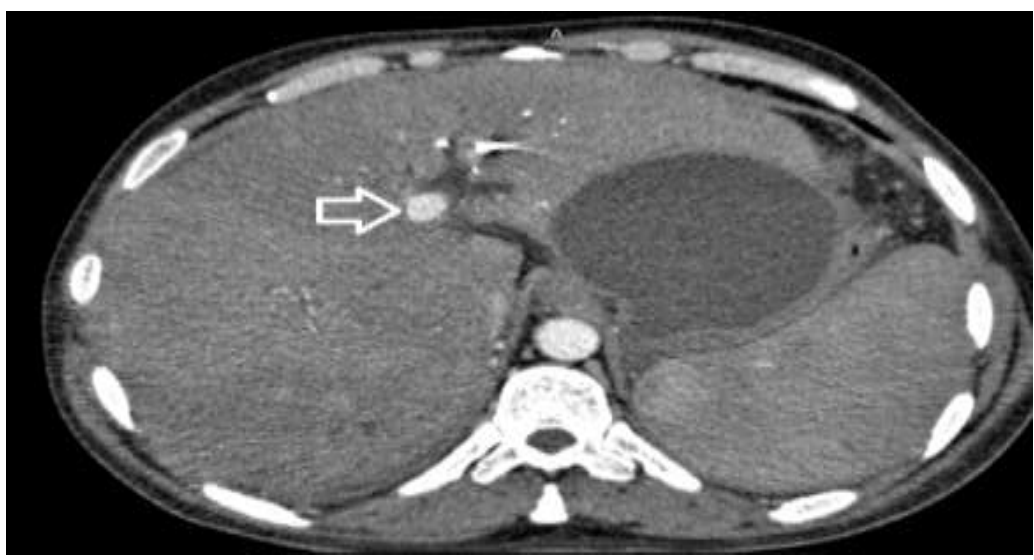

Figure (A) The arterial phase showed a saccular out pouching (pseudo-aneurysm) at the left hepatic artery

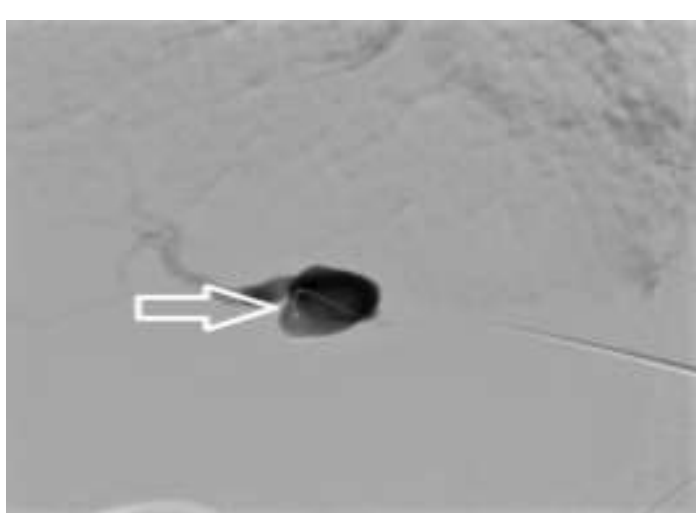

Figure (B) Selective angiogram of the hepatic artery clearly showed the pseudo-aneurysm (arrow

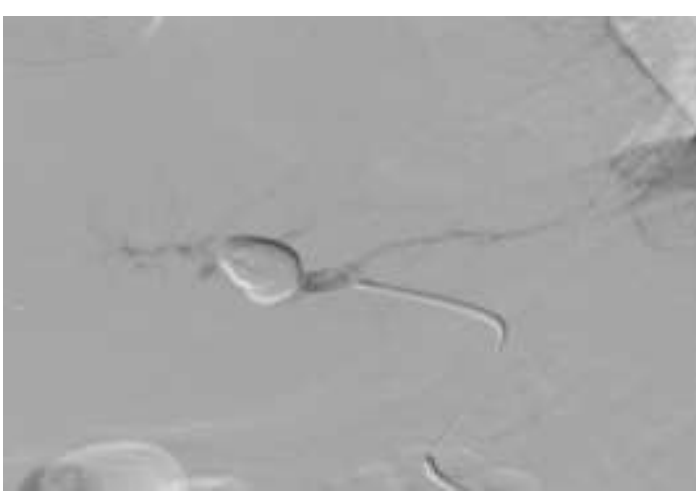

Figure(C) The pseudo-aneurysm was successfully managed using Histoacryl

Conclusions

Four radiologic imaging modalities are used in management of GI bleeding. Routine ultrasound; has the advantage of detecting large masses but has the disadvantage of not detecting small pseudoaneurysms, angiodysplasia, AVMs, small tumors or colonic tumors. Routine abdomen and pelvis CT with contrast has mainly the advantage of detecting tumors as a cause of bleeding while CTA has a better capability in detecting pseudoaneurysms, AVMs, active intraluminal extravasation and angiodysplasia. As regard catheter angiography its role was mainly therapeutic. CC-BY-NC 